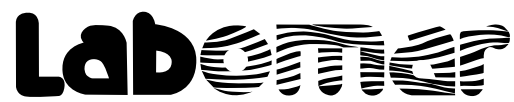

Arquivos de Ciências do Mar

\section{RELAÇÕES BIOMÉTRICAS DO GAMARÃO ROSA Penaeus subtilis (PÉREZ FARFANTE, 1967) NA PLATAFORMA CONTINENTAL AMAZÔNICA}

\author{
Biometric relationships of the brown shrimp \\ Penaeus subtilis (Pérez Farfante, 1967) in the \\ Amazon River continental shelf \\ José Augusto Negreiros Aragão ${ }^{1 *}$, Miguel Petrere Júnior², Israel Hidenburgo Aniceto Cintra ${ }^{3}$ \\ ${ }^{1}$ Analista Ambiental do Instituto Brasileiro do Meio Ambiente e dos Recursos Naturais Renováveis. \\ E-mail: j aragao@hotmail.com \\ 2 Professor Visitante Nacional Sênior da Universidade Federal de São Carlos. \\ E-mail: mpetrere@ufscar.br \\ ${ }^{3}$ Professor Adjunto IV do Instituto Socioambiental e dos Recursos Hídricos, Universidade Federal Rural da Amazônia \\ E-mail: israel.cintra@ufra.edu.br \\ ${ }^{*}$ Autor para correspondência
}

\begin{abstract}
RESUMO
No presente trabalho foram estimadas relações biométricas entre algumas medidas de comprimento e peso do corpo do camarão rosa Penaeus subtilis (Pérez Farfante, 1967) na plataforma continental amazônica. Foram utilizados dados de amostras obtidas a bordo de navio de pesquisa, bem como amostras obtidas por ocasião de desembarques nas empresas de pesca em Belém, estado do Pará, no período de 1995 a 1998. Para ajuste das equações foram utilizadas as técnicas de regressão linear e não linear baseadas nos métodos dos mínimos quadrados e máxima verossimilhança, respectivamente. Foram calculadas regressões para fêmeas e machos, considerando os dados de todo o período do estudo, agrupados numa base trimestral e anual. Verificou-se que as fêmeas crescem mais que os machos e, assim, as curvas das várias regressões para machos e fêmeas apresentaram diferenças significativas. Foram encontradas também diferenças expressivas entre os parâmetros aqui calculados e os parâmetros apresentados por outros autores em trabalhos anteriores. Os valores do fator de condição foram sempre superiores para os indivíduos maiores, sugerindo presença de indivíduos maduros ao longo de todo o ano, mas em menor proporção no primeiro trimestre.
\end{abstract}

Palavras-chave: Pesca do camarão; costa norte; relação peso-comprimento; regressão.

Recebido em: $27 / 3 / 2017$

Aprovado em: 11/9/2017

Publicado online em: 20/1/2018 


\section{ABSTRACT}

In the present paper biometrics relationships between measures of body length and body weight of the brow shrimp Penaeus subtilis (Pérez Farfante, 1967) in the Amazon River continental shelf, were estimated. Samples obtained on board of the research vessel Alm. Paulo Moreira as well as samples obtained on the landings at the fishing companies in Belém, from 1995 to 1998 were used in the analysis. The equations were fitted utilizing the linear and non linear regression techniques based on the least squares and maximum likelihood methods respectively. Regressions were estimated for females and males taking in account the data over the entire period, grouped by quarter and year. It was found that females are larger than males and significant differences between the curves for the several regressions for males and females were verified. Expressive differences between the parameters estimated here and the parameters presented by other authors in previous studies were also found. The values of the condition factor were always higher for larger individuals, suggesting the presence of mature individuals throughout the year, but to a lesser extend in the first trimester.

Key words: Shrimp fishery; Brazilian north coast; length-weight relationship; regression.

\section{INTRODUÇÃO}

A pesca industrial do camarão-rosa na costa norte do Brasil, constitui-se numa das atividades de maior importância no cenário pesqueiro regional e nacional, sendo eminentemente geradora de divisas. Belém, no estado do Pará, é o principal porto e base da indústria de processamento de camarão (Aragão et al., 2001). Algumas embarcações operam a partir de Fortaleza, no estado do Ceará, onde também existem indústrias de processamento e, nos anos 1980/1990, havia também embarcações que operavam com base em Macapá, no estado do Amapá (Aragão et al., 2004; Aragão et al., 2005).

A área onde se desenvolvem as pescarias fica compreendida entre a foz do rio Parnaíba (02 53'S), divisa dos estados do Piauí e Maranhão, e a foz do rio Oiapoque $\left(04^{\circ} 23^{\prime} \mathrm{N}\right)$, na fronteira com a Guiana Francesa, compreendendo a costa do Amapá, Pará e Maranhão (Figura 1). Esta área de pesca constitui parte de um extenso banco camaroneiro que se prolonga até as proximidades da foz do rio Orinoco, na Venezuela, cobrindo cerca de $223.000 \mathrm{~km}^{2}$ (IBAMA, 1994). Ressalta-se, no entanto, que hoje a frota concentra suas operações basicamente na costa dos estados do Pará e Amapá.

As capturas industriais de camarão-rosa na costa norte são compostas basicamente de Penaeus subtilis, com pequena ocorrência de Penaeus brasiliensis. Nos últimos anos, observou-se que a participação relativa do $P$. subtilis chega a representar praticamente a totalidade dos desembarques, conforme dados do Projeto Biologia e Pesca do Camarão-rosa na Região Norte do Brasil do CEPNOR (Aragão et al., 2001; Aragão et al., 2004).

Em geral, os exemplares capturados pela pesca industrial são descabeçados no mar e desembarcados na forma de cauda, não sendo possível tomar medidas como comprimento total ou comprimento de cefalotórax. Portanto, estabelecer relações entre as variáveis biométricas é de fundamental importância para os estudos de avaliação de estoques e outros estudos que envolvem composição das capturas por tamanho e peso. Relações biométricas são importantes, também, para se verificar diferenças morfométricas entre espécies e dimorfismo sexual em uma mesma espécie. 


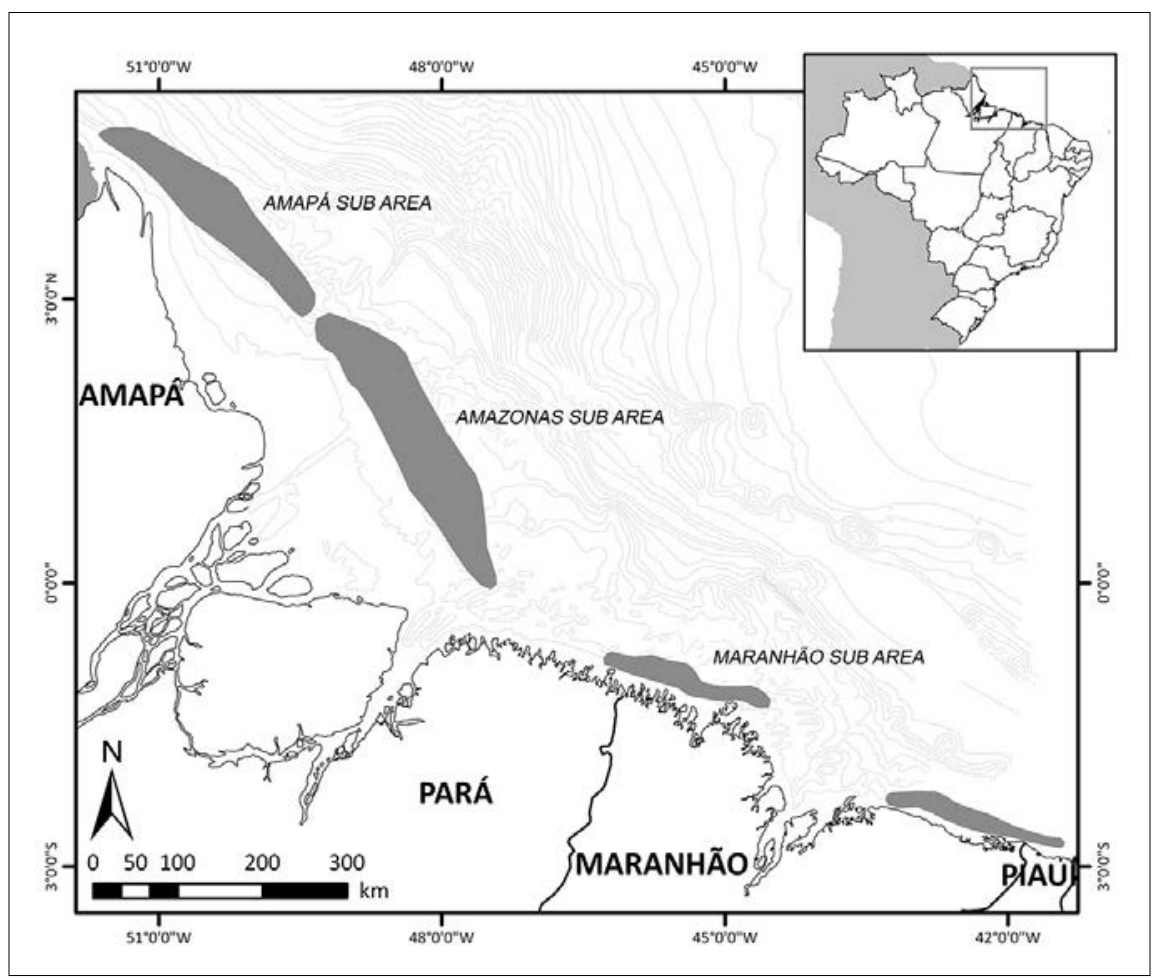

Figura 1 - Áreas de operação da pesca industrial de camarão-rosa Penaeus subtilis (Pérez Farfante, 1967) na costa norte do Brasil

Barbosa \& Rocha (1977), Dragovich et al. (1980) apresentaram equações descrevendo relações entre algumas variáveis biométricas do camarão-rosa por sexo, para a costa Norte do Brasil e para a costa Nordeste da América do Sul, respectivamente, em geral, numa base anual. As amostras utilizadas nas citadas análises eram, no entanto, relativamente pequenas, apresentavam faixas de comprimento limitadas e pouco representativas em termos temporais. Por outro lado, as medições, principalmente de peso, eram possivelmente bem menos acuradas que aquelas obtidas hoje, com equipamentos de maior precisão.

Assim, considerando-se a quantidade de amostras disponíveis no Laboratório de Crustáceos do CEPNOR, bem como a qualidade dos dados biométricos ali existentes, foram recalculadas algumas das relações estimadas pelos citados autores, com o objetivo de rever e refinar as estimativas dos parâmetros, bem como calculadas outras, ainda não disponíveis, tendo em vista a necessidade de se estabelecer relações confiáveis, utilizando os citados dados, para aplicar nos trabalhos de avaliação de estoque.

\section{MATERIAL E MÉTODOS}

Foram utilizados dados biométricos do camarão-rosa obtidos a partir de amostras de prospecções pesqueiras realizadas na costa norte do Brasil, coletadas em vários meses nos anos de 1996, 1997 e 1998, durante cruzeiros do Navio de Pesquisa Almirante Paulo Moreira, pertencente ao Centro de Pesquisa e Gestão dos Recursos Pesqueiros do Litoral Norte (CEPNOR/IBAMA). Utilizaram-se, também, dados de amostras realizadas por ocasião dos desembarques industriais nas empresas de pesca em Belém, Pará, no período de 1995 a 1998.

Durante o ano de 1996, foram realizadas expedições com o barco de pesquisa nos meses de agosto, setembro, outubro, novembro e dezembro. Em 1997, somente uma expe- 
dição foi realizada no mês de maio e, no ano de 1998, foram realizadas expedições nos meses de fevereiro, março, abril, maio e novembro. As amostras nos desembarques foram realizadas nos meses de setembro de 1995, fevereiro a julho de 1996 e março de 1998.

Durante os cruzeiros de pesquisa, uma vez finalizado cada arrasto, os camarões coletados eram devidamente acondicionados em caixas plásticas ("basquetas") com gelo e armazenados na câmara de refrigeração do navio, devidamente etiquetadas. Na chegada do barco, os camarões eram levados ao Laboratório de Crustáceos do CEPNOR, onde era feita a identificação da espécie, sexagem e biometria de todos os exemplares. Procedimento semelhante era ao adotado por ocasião dos desembarques comerciais, mas o trabalho era todo realizado no laboratório de controle de qualidade da própria planta de processamento.

As identificações específicas foram realizadas com o auxílio de várias publicações, entre estas, os trabalhos de Voss (1955), Fischer (1978), Holthuis (1980), Takeda (1983) e Williams (1984). As medidas tomadas durante as amostragens estão descritas a seguir e ilustradas na Figura 2: Comprimento total $(L t)$ - distância linear, ao milímetro inferior, tomada do extremo distal do rostro ao extremo posterior do telson; Comprimento da cauda ou abdômen $(L c)$ - distância linear, ao milímetro inferior, tomada do extremo anterior do primeiro segmento abdominal ao extremo posterior do telson; Peso total $(W t)$ - peso total do indivíduo em gramas, com precisão de $0,01 \mathrm{~g}$; Peso da cauda ou abdômen $(W c)$ - peso da cauda ou abdômen do indivíduo em gramas, com precisão de 0,01 g.

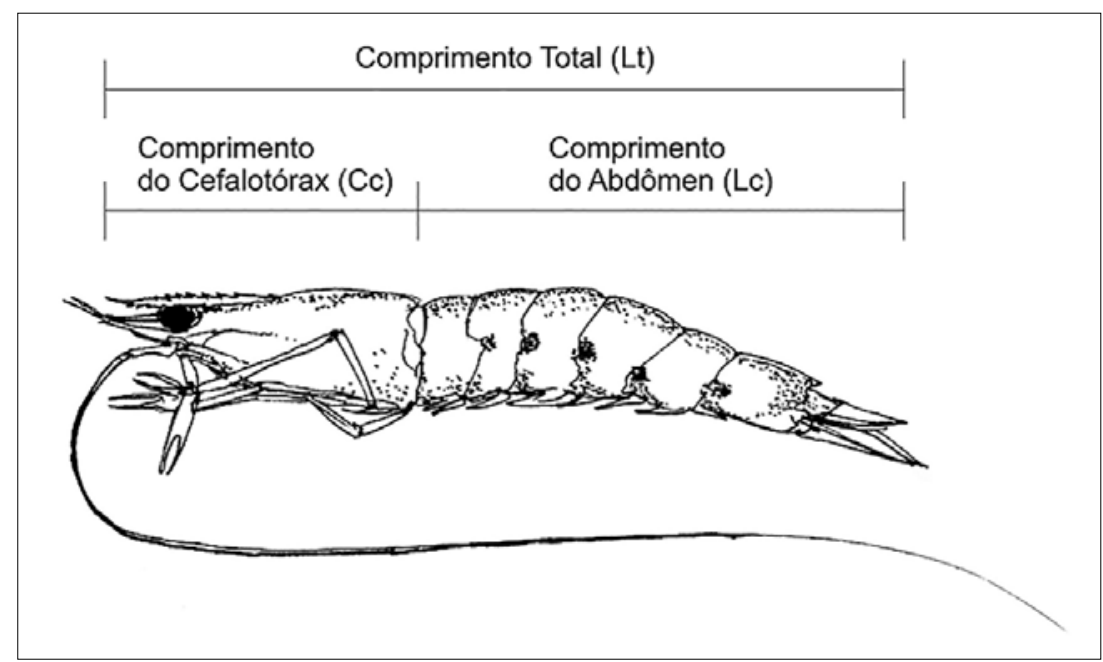

Figura 2 - Ilustração das medidas morfométricas tomadas nas amostras de camarão-rosa da costa norte do Brasil

As medidas de comprimento foram tomadas com paquímetro de aço (0 a $250 \mathrm{~mm}$ ), com precisão de $0,01 \mathrm{~mm}$, com os indivíduos esticados, com a parte ventral gentilmente pressionada sobre uma superfície plana. A pesagem foi feita após a drenagem do excesso de água no corpo do animal, com papel toalha, e o peso correspondente obtido com uma balança semi-analítica, com precisão de 0,01 g. Os dados das amostras foram sistematizados e digitados em planilhas eletrônicas e, a partir daí, o tratamento dos dados e as análises foram conduzidas por meio do software livre denominado "R Development Core Team, 2009" (http://cran.r-project.org/web/packages/nlrwr/ index.html).

Antes de se proceder ao ajuste das regressões, foi realizada uma análise exploratória nos dados, em busca de erros grosseiros ou claros equívocos de digitação. Isto pôde ser 
feito com certa facilidade, por meio de gráficos de dispersão para cada par de medidas cuja relação seria determinada e da comparação simultânea das quatro medidas tomadas (comprimento total, comprimento de cauda, peso total e peso de cauda). Por meio deste processo foi possível identificar, corrigir e excluir observações claramente inconsistentes, atribuídas a erros de mensuração e, na maioria dos casos, evidentes equívocos de digitação. Concluído o processo, estimaram-se estatísticas descritivas, incluindo média, valores máximos e valores mínimos e desvio padrão para as variáveis de cada sexo.

As relações consideradas no presente trabalho foram: peso total (g) versus comprimento total $(\mathrm{cm})$; peso de cauda $(\mathrm{g})$ versus comprimento de cauda $(\mathrm{cm})$; peso total $(\mathrm{g})$ versus peso de cauda $(\mathrm{g})$; e comprimento total $(\mathrm{cm})$ versus comprimento de cauda $(\mathrm{g})$. Para efeito de estimação, foram consideradas as amostras agrupadas por trimestre e para todo o período da coleta dos dados, uma vez que não houve regularidade de coleta durante todos os meses em cada um dos quatro anos de realização da pesquisa. Cada trimestre do ano corresponde aproximadamente a uma das fases do ciclo hidrológico da região: período de aumento da vazão do rio (janeiro a março); período de cheia do rio (abril a junho); período de diminuição da vazão do rio (julho a setembro); e período de seca do rio (outubro a dezembro).

Os gráficos de dispersão dos dados aqui analisados sugerem, como era de se esperar, que uma função potência, com dois parâmetros e um termo de erro multiplicativo, deva ser utilizada para modelar a relação peso versus comprimento, ressaltando-se que a descrição desta relação por esta função está bem fundamentada em bases biológicas. Para ajuste das curvas, utilizou-se o modelo $W=a L^{b} e$, onde $a$ e $b$ são constantes e $\varepsilon$ é o termo aleatório ou resíduo (Ricker, 1975; Sampson, 2008; Ogle, 2009). As relações comprimento total versus comprimento de cauda e peso total versus peso de cauda são descritas por uma função linear do tipo $y=a+b x$ e o ajuste foi feito por meio do modelo de regressão linear simples. Os modelos utilizados para cálculo das relações foram:

- Relação peso total versus comprimento total: $W t=a L t^{b} e^{\varepsilon}$

- Relação peso de cauda versus comprimento de cauda: $W_{c}=a L c^{b} e^{\varepsilon}$

- Relação peso total versus peso de cauda: $W t=a+b W_{c}+\varepsilon$

- Relação comprimento total versus comprimento de cauda: $L t=a+b L c+\varepsilon$

A estimação dos parâmetros das regressões para o modelo potência foi feita utilizando-se a técnica de regressão não linear, uma vez que a função que as descreve é não linear em relação ao parâmetro $\beta$. O melhor ajuste dos dados à curva é obtido minimizando-se a soma dos quadrados dos resíduos $(S Q R)$ em relação às estimativas dos parâmetros:

$$
\operatorname{SQR}(\beta)=\sum_{i=1}^{n}\left[y_{i}-f\left(x_{i}, \beta\right)\right]^{2}
$$

Diferentemente da regressão linear, para se obter a minimização da soma dos quadrados $(S Q R)$ e a consequente estimação dos parâmetros são necessários métodos numéricos iterativos de otimização. Uma vez estimados os parâmetros $(\beta)$, a variância residual estimada é dada por $s^{2}=\frac{S Q R}{n-p}$, onde $n$ é o número de observações e $P$ o número de parâmetros (Fox, 2002).

Como é comum em relações que envolvem medidas de peso e comprimento, a variância da variável resposta (peso) aumenta à medida que crescem os valores da variável 
explanatória (comprimento), ou seja, a variância é heterocedástica. Também, nem sempre os resíduos são normalmente distribuídos. Estes problemas foram remediados aplicando-se uma transformação a ambos os lados da equação, o que preserva a relação original entre as variáveis (Ritz \& Streibig, 2008):

$$
y=f(x, \beta) \quad h(y)=h[f(x, \beta)]
$$

No caso da relação peso versus comprimento, por exemplo, teríamos:

$$
W t=a L^{b}{ }_{t} \quad W t=f\left[L_{t},(a, b)\right] \quad h\left\{f\left[L_{t},(a, b)\right]\right\}=h\left[a L^{b}{ }_{t}\right]+\varepsilon
$$

Para se determinar a melhor transformação a ser aplicada aos dois lados da equação foi utilizado o método Box-Cox (Box \& Cox, 1964, apud Sokal \& Rohlf, 1995; Ritz \& Streibig, 2008) definido da seguinte maneira:

$$
h_{\lambda}= \begin{cases}\frac{y^{\lambda}-1}{\lambda} & \lambda \neq 0 \\ \log (y) & \lambda=0\end{cases}
$$

O método permite que se encontre o parâmetro da transformação potência $(\lambda)$ que maximiza a máxima verossimilhança ("likelihood"), quando um conjunto determinado de variáveis explanatórias é ajustado a $\frac{\mathrm{y}^{\lambda}-1}{\lambda}$ como variável resposta. Quando $\lambda$ é igual a 0, obtém-se a transformação logarítmica. Nem sempre o resultado funciona, sendo necessária nova avaliação da normalidade dos resíduos após a transformação (Ritz \& Streibig, 2008).

O processo de transformação Box-Cox e ajuste das regressões foi realizado com a função $n l s($ ) do software livre $R$, que utiliza o algoritmo de Gauss-Newton para determinar os parâmetros que minimizam $S Q R$ e oferecem o melhor ajuste, e com a função boxcox.nls() do pacote estatístico denominado nlrwr (Ritz \& Streibig, 2008), também integrante do $R$. Nas análises do presente trabalho, o parâmetro $\lambda$ da transformação Box-Cox foi determinado por meio da regressão calculada para os dados com os sexos agrupados e o mesmo valor utilizado para o cálculo da regressão de fêmeas e machos separados, de forma que as curvas pudessem ser comparadas.

Uma vez que a função $n l s()$ está fundamentada em processos iterativos, ela requer valores iniciais para proceder a estimação dos parâmetros. Estes valores foram obtidos por meio do ajuste linear tradicional, com transformação logarítmica, e alimentaram a função por ocasião do ajuste preliminar. No passo seguinte, os parâmetros finais foram determinados com a função boxcox.nls() e os resíduos reavaliados graficamente. Foram então elaborados gráficos dos dados brutos sobrepostos pelas curvas ajustadas. Foram calculadas inicialmente regressões com os dados dos cruzeiros de pesquisa e de desembarque separados e depois com os dados agrupados.

Os pressupostos da análise de regressão, ou seja, o padrão de normalidade da distribuição dos resíduos, a homogeneidade de variâncias e a independência dos dados, foram validados por meio de gráficos de resíduos e resíduos padronizados versus valores previstos e por meio de gráficos de probabilidades normais (Ragonese et al., 1987; Crawler, 2007; Venables \& Simth, 2008; Ogle, 2009).

Para estimação dos parâmetros da relação peso total versus peso de cauda, embora o modelo utilizado tenha sido linear $\left(W_{t}=a+b W_{c}+\varepsilon\right.$, os cálculos foram feitos utilizando-se também a função $n l s()$, uma vez que os dados apresentam variância heterocedástica, com 
resíduos no formato típico de corneta. Os parâmetros da relação peso total versus comprimento total foram estimados também com a unidade de comprimento em centímetros para permitir comparação com as estimativas de outros autores.

Diferenças entre as curvas para os dados de machos e fêmeas com os dados agrupados para todo o período foram avaliadas por meio da "análise da soma dos quadrados dos resíduos (ARSS)", utilizando-se a seguinte expressão (Haddon, 2011):

$$
F=\frac{\frac{R S S_{p}-\Sigma R S S_{i}}{D F_{p}-\Sigma D F_{i}}}{\frac{\Sigma R S S_{i}}{\Sigma D F_{i}}}=\frac{\frac{R S S_{p}-\Sigma R S S_{i}}{3(K-1)}}{\frac{\Sigma R S S_{i}}{N-3 K}}
$$

Onde a estatística $F$ é determinada com $3(K-1)$ e $N-3 K$ graus de liberdade, $K$ é o número de curvas sendo comparadas e $N$ é o tamanho das amostras agrupadas. Ou seja, o princípio do teste envolve a comparação da soma dos quadrados dos resíduos $\left(R S S_{p}\right)$ para a hipótese de que todas as curvas são coincidentes e, neste caso, cada conjunto de dados seria efetivamente uma amostra da mesma população, contra o resultado da soma dos quadrados dos resíduos $\left(\Sigma R S S_{i}\right)$ da hipótese que os conjuntos de dados se originam de populações independentes. Este tipo de teste pode ser aplicado a qualquer tipo de curvas (Ritz \& Streibig, 2008; Haddon, 2011).

A partir das relações trimestrais de peso total versus comprimento total foi determinado o fator de condição para cada classe de comprimento, dentro de cada período, e procurou-se relacionar diferenças no padrão de cada um deles com as fases do ciclo de vida da espécie. O fator de condição médio por classe de comprimento, em cada estação do ano, derivado do fator de condição de Fulton, onde $\mathrm{K}=100 \frac{\mathrm{W}}{L^{3}}$, (Froese, 2006), foi estimado utilizando-se os parâmetros das equações sazonais de peso-comprimento para o camarão rosa:

$$
K_{\text {médio }}=100 * \mathrm{a} * L^{b-3}
$$

Comparações das alterações sazonais na condição fisiológica dos indivíduos foram feitas então por meio de um gráfico do logaritmo do fator de condição por faixa de comprimento versus o logaritmo do comprimento, cuja tendência aproximada é de uma linha reta (Froese, 2006).

\section{RESULTADOS}

Um total de 7190 indivíduos foi amostrado em todo o período e utilizado nas análises, sendo 3674 fêmeas e 3516 machos. Nos desembarques, foram amostrados 3057 indivíduos, com 1937 fêmeas e 1120 machos, enquanto 4133 foram obtidos a bordo do navio de pesquisa, com 1737 fêmeas e 2396 machos (Tabela 1).

Estatísticas descritivas, contemplando valores mínimos, médios, máximos e desvio padrão observados, para comprimento total $(L t)$, comprimento de cauda $(L c)$, peso total $(W t)$ e peso de cauda $(W c)$, para cada sexo, com as amostras do $P$. subtilis agrupadas para todo o período, são apresentados na Tabela 2.

Nas Tabelas 3, 4, 5 e 6 são apresentados os parâmetros estimados para as equações que descrevem as relações entre as variáveis biométricas aqui analisadas. Estas foram calculadas com base nas amostras obtidas em cruzeiros de pesquisa e desembarques, agrupadas por trimestre e para todo o período, bem como os valores mínimos, médios e máximos das medidas utilizadas na regressão. 
Tabela 1 - Número de indivíduos por sexo nas amostragens biométricas de Penaeus subtilis obtidas nos desembarques industriais e em cruzeiros do navio de pesquisa Almirante Paulo Moreira, na plataforma continental amazônica, no período de 1995 a 1998.

\begin{tabular}{|c|c|c|c|c|c|c|c|c|c|c|c|c|c|}
\hline Anos & Local da amostragem & Sexo & Fev & Mar & Abr & Mai & Jun & Jul & Ago & Set & Out & Nov & Total \\
\hline \multirow[t]{2}{*}{1995} & Empresa de pesca & Fêmea & & & & & & & & 412 & & & 412 \\
\hline & & Macho & & & & & & & & 401 & & & 401 \\
\hline \multirow[t]{2}{*}{1996} & Empresa de pesca & Fêmea & 39 & 49 & 227 & 125 & 495 & 83 & & & & & 1018 \\
\hline & & Macho & 16 & 24 & 6 & 117 & 279 & 17 & & & & & 459 \\
\hline \multirow[t]{4}{*}{1998} & Empresa de pesca & Fêmea & & 507 & & & & & & & & & 507 \\
\hline & & Macho & & 260 & & & & & & & & & 260 \\
\hline & Subtotal & Fêmea & 39 & 556 & 227 & 125 & 495 & 83 & 0 & 412 & 0 & 0 & 1937 \\
\hline & & Macho & 16 & 284 & 6 & 117 & 279 & 17 & 0 & 401 & 0 & 0 & 1120 \\
\hline \multirow[t]{2}{*}{1996} & NPq.Paulo Moreira & Fêmea & & & & & & 71 & 155 & 131 & 219 & 97 & 673 \\
\hline & & Macho & & & & & & 27 & 108 & 210 & 178 & 120 & 643 \\
\hline \multirow[t]{2}{*}{1997} & NPq.Paulo Moreira & Fêmea & & & & 313 & & & & & & & 313 \\
\hline & & Macho & & & & 690 & & & & & & & 690 \\
\hline \multirow[t]{7}{*}{1998} & NPq.Paulo Moreira & Fêmea & 425 & 10 & 39 & 277 & & & & & & & 751 \\
\hline & & Macho & 639 & 14 & 126 & 284 & & & & & & & 1063 \\
\hline & Subtotal & Fêmea & 425 & 10 & 39 & 590 & 0 & 71 & 155 & 131 & 219 & 97 & 1737 \\
\hline & & Macho & 639 & 14 & 126 & 974 & 0 & 27 & 108 & 210 & 178 & 120 & 2396 \\
\hline & Total geral & Fêmea & 464 & 566 & 266 & 715 & 495 & 154 & 155 & 543 & 219 & 97 & 3674 \\
\hline & & Macho & 655 & 298 & 132 & 1091 & 279 & 44 & 108 & 611 & 178 & 120 & 3516 \\
\hline & & Total & 1119 & 864 & 398 & 1806 & 774 & 198 & 263 & 1154 & 397 & 217 & 7190 \\
\hline
\end{tabular}

Tabela 2 - Valores máximos, médios e mínimos de medidas biométricas de $P$. subtilis, obtidas em amostras de cruzeiros de pesquisa na plataforma continental amazônica e em desembarques da frota industrial, no período de 1995 a 1998.

\begin{tabular}{|c|c|c|c|c|c|c|c|c|c|c|c|c|}
\hline \multirow{3}{*}{$\begin{array}{c}\text { Medida } \\
\text { Sexo } \\
\text { (n) }\end{array}$} & \multicolumn{3}{|c|}{ Lt (mm) } & \multicolumn{3}{|c|}{$\mathrm{Lc}(\mathrm{mm})$} & \multicolumn{3}{|c|}{ Wt (g) } & \multicolumn{3}{|c|}{ Wc (g) } \\
\hline & Fêmea & Macho & Geral & Fêmea & Macho & Geral & Fêmea & Macho & Geral & Fêmea & Macho & Geral \\
\hline & 3328 & 3153 & 6481 & 3328 & 3153 & 6481 & 2920 & 2702 & 5622 & 2920 & 2702 & 5622 \\
\hline Mínimo & 60,0 & 71,0 & 60,0 & 40,0 & 46,0 & 40,0 & 1,2 & 3,0 & 1,2 & 0,8 & 2,0 & 0,8 \\
\hline Média & 144,2 & 124,7 & 134,7 & 92,0 & 80,4 & 86,3 & 21,9 & 12,2 & 17,2 & 13,5 & 8,0 & 10,9 \\
\hline Máximo & 232,0 & 195,0 & 232,0 & 152,0 & 129,0 & 152,0 & 98,6 & 47,5 & 98,6 & 57,8 & 30,3 & 57,8 \\
\hline Desv. pad. & 27,3 & 14,5 & 24,1 & 17,7 & 9,7 & 15,5 & 13,7 & 4,8 & 11,5 & 7,9 & 3,1 & 6,7 \\
\hline
\end{tabular}

\begin{tabular}{|c|c|c|c|c|c|c|}
\hline \multirow{2}{*}{ Sexo } & \multirow{2}{*}{ Parâmetro } & \multicolumn{4}{|c|}{ Trimestre } & \multirow{2}{*}{$\begin{array}{c}\text { Dados } \\
\text { agrupados }\end{array}$} \\
\hline & & I & II & III & IV & \\
\hline \multirow{11}{*}{ Fêmea } & $(n)$ & 1013 & 1146 & 225 & 855 & 3239 \\
\hline & MaxLt & 19,7 & 21,4 & 21,4 & 21,5 & 21,5 \\
\hline & MedLt & 14,0 & 15,3 & 13,8 & 13,3 & 14,3 \\
\hline & MinLt & 6,4 & 7,0 & 8,0 & 6,0 & 6,0 \\
\hline & MaxLc & 12,6 & 13,7 & 13,6 & 13,9 & 13,9 \\
\hline & MedLc & 8,9 & 9,8 & 8,8 & 8,5 & 9,1 \\
\hline & MinLc & 4,0 & 4,4 & 5,0 & 4,0 & 4,0 \\
\hline & $a$ & 0,212808 & 0,170030 & 0,126140 & 0,753354 & 0,465716 \\
\hline & d.p.(a) & 0,072051 & 0,067160 & 0,140620 & 0,054800 & 0,033388 \\
\hline & b & 1,551 & 1,546 & 1,547 & 1,478 & 1,516 \\
\hline & d.p.(b) & 0,008 & 0,007 & 0,017 & 0,007 & 0,004 \\
\hline \multirow{11}{*}{ Macho } & $(\mathrm{n})$ & 941 & 1204 & 130 & 847 & 3122 \\
\hline & MaxLt & 15,9 & 19,0 & 17,1 & 16,7 & 19,0 \\
\hline & MedLt & 12,2 & 12,7 & 12,1 & 12,4 & 12,4 \\
\hline & MinLt & 8,0 & 7,7 & 8,8 & 8,5 & 7,7 \\
\hline & MaxLC & 10,5 & 12,7 & 11,1 & 11,2 & 12,7 \\
\hline & MedLc & 7,9 & 8,2 & 7,8 & 8,0 & 8,0 \\
\hline & MinLc & 5,0 & 5,0 & 5,7 & 5,2 & 5,0 \\
\hline & a & 1,048550 & 0,798630 & 0,725210 & 0,919500 & 0,869721 \\
\hline & d.p.(a) & 0,109030 & 0,090540 & 0,128950 & 0,079460 & 0,050814 \\
\hline & b & 1,418 & 1,455 & 1,456 & 1,431 & 1,442 \\
\hline & d.p.(b) & 0,014 & 0,011 & 0,017 & 0,010 & 0,006 \\
\hline
\end{tabular}

Tabela 3 - Parâmetros estimados para regressões peso total $(\mathrm{g})$ versus comprimento total $(\mathrm{cm})$ de $P$. subtilis na plataforma continental amazônica. 
Tabela 4 - Parâmetros estimados para regressões peso cauda (g) versus comprimento cauda (cm) de P. subtilis na plataforma continental amazônica.

\begin{tabular}{|c|c|c|c|c|c|c|}
\hline \multirow{2}{*}{ Sexo } & \multirow{2}{*}{ Parâmetro } & \multicolumn{4}{|c|}{ Trimestre } & \multirow{2}{*}{$\begin{array}{c}\text { Dados } \\
\text { agrupado }\end{array}$} \\
\hline & & 1 & II & III & IV & \\
\hline \multirow{11}{*}{ Fêmea } & (n) & 1017 & 1462 & 225 & 443 & 3147 \\
\hline & MaxLc & 12,6 & 13,7 & 13,6 & 13,8 & 13,8 \\
\hline & MedLc & 8,9 & 9,9 & 8,8 & 7,8 & 9,2 \\
\hline & MinLc & 4,0 & 4,4 & 5,0 & 4,0 & 4,0 \\
\hline & MaxWc & 32,0 & 38,1 & 39,5 & 44,8 & 44,8 \\
\hline & MedWc & 11,6 & 15,9 & 11,4 & 8,8 & 13,2 \\
\hline & MinWc & 1,0 & 1,4 & 1,9 & 0,8 & 0,8 \\
\hline & $a$ & 0,015411 & 0,017317 & 0,011886 & 0,014443 & 0,014876 \\
\hline & d.p.(a) & 0,000787 & 0,000504 & 0,000727 & 0,000556 & 0,000302 \\
\hline & $\mathrm{b}$ & 2,997 & 2,955 & 3,107 & 3,024 & 3,016 \\
\hline & d.p.(b) & 0,023 & 0,013 & 0,028 & 0,018 & 0,009 \\
\hline \multirow{11}{*}{ Macho } & (n) & 933 & 1436 & 134 & 443 & 2946 \\
\hline & MaxLC & 10,5 & 12,7 & 11,1 & 10,7 & 12,7 \\
\hline & MedLc & 7,9 & 8,3 & 7,8 & 7,8 & 1,0 \\
\hline & MinLc & 5,0 & 5,0 & 5,7 & 5,2 & 5,0 \\
\hline & MaxWc & 16,1 & 30,3 & 19,5 & 18,5 & 30,3 \\
\hline & MedWc & 7,6 & 8,9 & 7,1 & 7,2 & 8,1 \\
\hline & MinWc & 2,3 & 2,0 & 2,6 & 2,1 & 2,0 \\
\hline & $a$ & 0,018030 & 0,019325 & 0,017076 & 0,015199 & 0,017614 \\
\hline & d.p.(a) & 0,001070 & 0,000852 & 0,001199 & 0,000705 & 0,000496 \\
\hline & $b$ & 2,916 & 2,878 & 2,911 & 2,974 & 2,920 \\
\hline & d.p.(b) & 0,029 & 0,021 & 0,034 & 0,022 & 0,013 \\
\hline
\end{tabular}

d. p. - desvio padrão

Tabela 5 - Parâmetros estimados para regressões peso total (g) versus peso de cauda $(\mathrm{g})$ de $P$. subtilis na plataforma continental amazônica.

\begin{tabular}{|c|c|c|c|c|c|c|}
\hline \multirow{2}{*}{ Sexo } & \multirow{2}{*}{ Parâmetro } & \multicolumn{4}{|c|}{ Trimestre } & \multirow{2}{*}{$\begin{array}{c}\text { Dados } \\
\text { agrupados }\end{array}$} \\
\hline & & 1 & II & III & IV & \\
\hline \multirow{11}{*}{ Fêmea } & (n) & 1014 & 1142 & 226 & 444 & 2826 \\
\hline & MaxWt & 52,2 & 68,9 & 70,9 & 68,1 & 70,9 \\
\hline & MedWt & 18,9 & 25,5 & 18,2 & 13,1 & 20,6 \\
\hline & MinWt & 1,7 & 2,1 & 3,1 & 1,2 & 1,2 \\
\hline & MaxWc & 32,0 & 38,1 & 39,5 & 44,8 & 44,8 \\
\hline & MedWc & 11,6 & 15,6 & 11,4 & 8,7 & 12,7 \\
\hline & MinWc & 1,0 & 1,4 & 1,9 & 0,8 & 0,8 \\
\hline & $a$ & $-0,079538$ & $-0,867591$ & $-0,477640$ & $-0,026685$ & $-0,315129$ \\
\hline & d.p.(a) & 0,036584 & 0,043403 & 0,088660 & 0,023418 & 0,017865 \\
\hline & $b$ & 1,633 & 1,672 & 1,633 & 1,482 & 1,624 \\
\hline & d.p.(b) & 0,005 & 0,005 & 0,012 & 0,007 & 0,003 \\
\hline \multirow{11}{*}{ Macho } & (n) & 942 & 1166 & 135 & 452 & 2695 \\
\hline & MaxWt & 25,6 & 47,5 & 32,2 & 28,9 & 47,5 \\
\hline & MedWt & 11,8 & 13,2 & 10,8 & 10,6 & 12,2 \\
\hline & MinWt & 3,8 & 3,1 & 3,9 & 3,0 & 3,0 \\
\hline & MaxWc & 16,1 & 30,3 & 19,5 & 18,5 & 30,3 \\
\hline & MedWc & 7,6 & 8,7 & 7,1 & 7,2 & 8,0 \\
\hline & MinWc & 2,3 & 2,0 & 2,6 & 2,1 & 2,0 \\
\hline & $a$ & 0,438718 & $-0,359860$ & $-0,089010$ & $-0,012280$ & $-0,076340$ \\
\hline & d.p.(a) & 0,060741 & 0,047490 & 0,118320 & 0,041210 & 0,030040 \\
\hline & $b$ & 1,497 & 1,554 & 1,521 & 1,466 & 1,529 \\
\hline & d.p.(b) & 0,009 & 0,007 & 0,021 & 0,008 & 0,005 \\
\hline
\end{tabular}

d. p. - desvio padrão 


\begin{tabular}{|c|c|c|c|c|c|c|}
\hline \multirow{2}{*}{ Sexo } & \multirow{2}{*}{ Parâmetro } & \multicolumn{4}{|c|}{ Trimestre } & \multirow{2}{*}{$\begin{array}{c}\text { Dados } \\
\text { agrupados }\end{array}$} \\
\hline & & 1 & II & III & IV & \\
\hline \multirow{11}{*}{ Fêmea } & (n) & 1013 & 1146 & 225 & 855 & 3239 \\
\hline & MaxLt & 19,7 & 21,4 & 21,4 & 21,5 & 21,5 \\
\hline & MedLt & 14,0 & 15,3 & 13,8 & 13,3 & 14,3 \\
\hline & MinLt & 6,4 & 7,0 & 8,0 & 6,0 & 6,0 \\
\hline & MaxLc & 12,6 & 13,7 & 13,6 & 13,9 & 13,9 \\
\hline & MedLc & 8,9 & 9,8 & 8,8 & 8,5 & 9,1 \\
\hline & MinLc & 4,0 & 4,4 & 5,0 & 4,0 & 4,0 \\
\hline & $a$ & 0,212808 & 0,170030 & 0,126140 & 0,753354 & 0,465716 \\
\hline & d.p.(a) & 0,072051 & 0,067160 & 0,140620 & 0,054800 & 0,033388 \\
\hline & b & 1,551 & 1,546 & 1,547 & 1,478 & 1,516 \\
\hline & d.p.(b) & 0,008 & 0,007 & 0,017 & 0,007 & 0,004 \\
\hline \multirow{11}{*}{ Macho } & (n) & 941 & 1204 & 130 & 847 & 3122 \\
\hline & MaxLt & 15,9 & 19,0 & 17,1 & 16,7 & 19,0 \\
\hline & MedLt & 12,2 & 12,7 & 12,1 & 12,4 & 12,4 \\
\hline & MinLt & 8,0 & 7,7 & 8,8 & 8,5 & 7,7 \\
\hline & MaxLc & 10,5 & 12,7 & 11,1 & 11,2 & 12,7 \\
\hline & MedLc & 7,9 & 8,2 & 7,8 & 8,0 & 8,0 \\
\hline & MinLc & 5,0 & 5,0 & 5,7 & 5,2 & 5,0 \\
\hline & $a$ & 1,048550 & 0,798630 & 0,725210 & 0,919500 & 0,869721 \\
\hline & d.p.(a) & 0,109030 & 0,090540 & 0,128950 & 0,079460 & 0,050814 \\
\hline & b & 1,418 & 1,455 & 1,456 & 1,431 & 1,442 \\
\hline & d.p.(b) & 0,014 & 0,011 & 0,017 & 0,010 & 0,006 \\
\hline
\end{tabular}

Tabela 6 - Parâmetros estimados para regressões comprimento total $(\mathrm{cm})$ versus comprimento cauda $(\mathrm{cm})$ de $P$. subtilis na plataforma continental amazônica.

Os valores das estimativas do parâmetro $b$ nas regressões peso total versus comprimento total são todos numericamente um pouco maiores que 3, o que tradicionalmente é interpretado como crescimento moderadamente alométrico da espécie.

Um resumo das estimativas dos parâmetros das equações calculadas para as relações biométricas com os dados do $P$. subtilis agrupados para todo o período e os resultados dos testes de comparação das curvas é apresentado na Tabela 7.

Tabela 7 - Parâmetros estimados para as relações biométricas anuais do camarão-rosa ( $P$. subtilis) na plataforma continental amazônica e resultado dos testes de comparação de igualdade de curvas para as regressões.

\begin{tabular}{|c|c|c|c|c|c|c|c|}
\hline Relação & Sexo & $\mathbf{n}$ & $\lambda$ & a & b & $\mathbf{F}$ & $p$ \\
\hline \multirow{2}{*}{$\mathrm{Wt} \sim \mathrm{Lt}$} & Fêmea & 2828 & \multirow{2}{*}{0,3} & 0,00316 & 3,263 & \multirow{2}{*}{43,2} & \multirow{2}{*}{$0,00 E+O$} \\
\hline & Macho & 2684 & & 0,00460 & 3,111 & & \\
\hline \multirow{2}{*}{$\mathrm{Wc} \sim \mathrm{Lc}$} & Fêmea & 3147 & \multirow{2}{*}{0,2} & 0,01488 & 3,016 & \multirow{2}{*}{130,0} & \multirow{2}{*}{$0,00 E+O$} \\
\hline & Macho & 2946 & & 0,01761 & 2,920 & & \\
\hline \multirow{2}{*}{$\mathrm{Lt} \sim \mathrm{LC}$} & Fêmea & 2826 & & $-0,31513$ & 1,624 & \multirow{2}{*}{237,8} & \multirow{2}{*}{$0,00 E+00$} \\
\hline & Macho & 2695 & & $-0,07634$ & 1,529 & & \\
\hline \multirow{2}{*}{$\mathrm{Wt} \sim \mathrm{Wc}$} & Fêmea & 3239 & & 0,46572 & 1,516 & \multirow{2}{*}{272,3} & \multirow{2}{*}{$0,00 E+00$} \\
\hline & Macho & 3122 & & 0,86972 & 1,442 & & \\
\hline
\end{tabular}

Por meio da "análise da soma dos quadrados dos resíduos (ARSS)", verifica-se que as curvas para todas as regressões apresentam diferenças altamente significativas entre machos e fêmeas. Gráficos de dispersão dos valores observados com as curvas ajustadas para todas as relações, calculadas com os dados agrupados, são apresentados nas Figuras 3 a 6, ficando também visualmente evidente as diferenças entre as curvas para machos e fêmeas. Deve-se notar nestas figuras que a vírgula (,) corresponde ao ponto (.) que é o padrão ("default") do programa $R$. 
Figura 3 - Relação peso total versus comprimento total para fêmeas e machos do P. subtilis na plataforma continental amazônica.
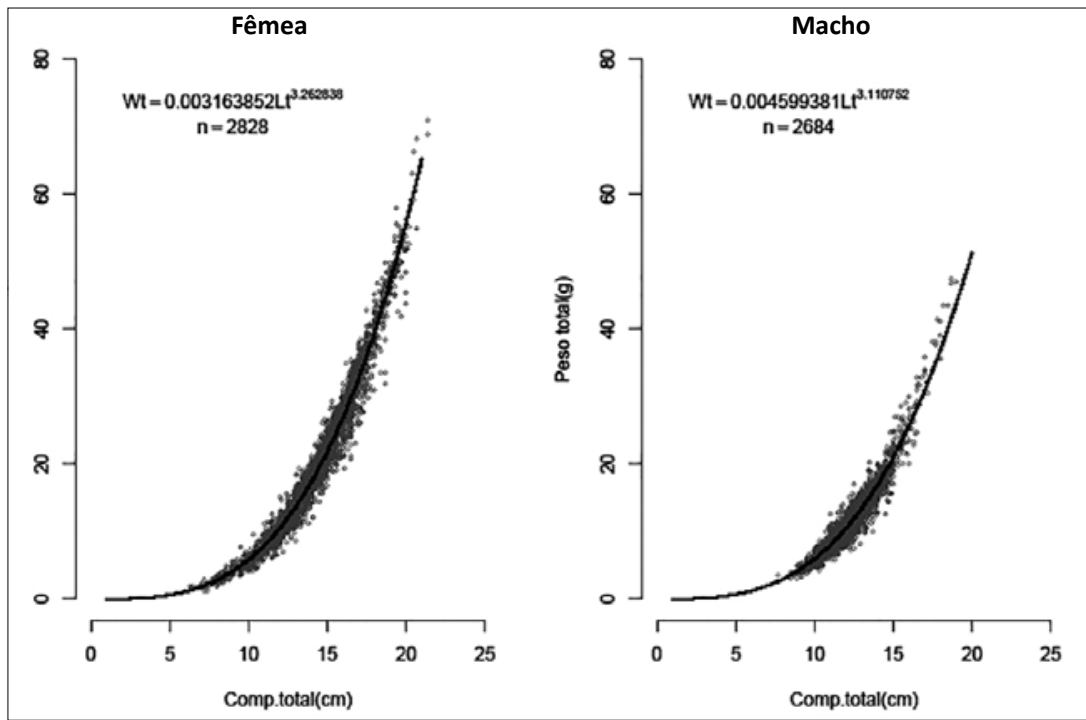

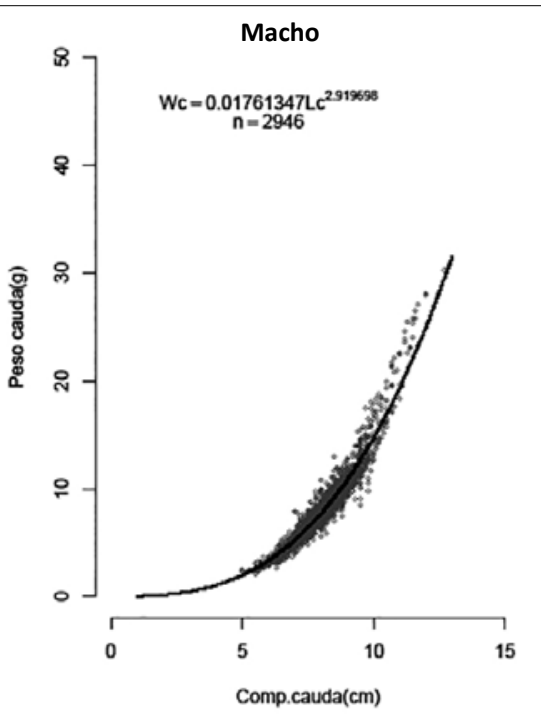

Figura 4 - Relação peso cauda versus comprimento de cauda para fêmeas e machos do $P$. subtilis na plataforma continental amazônica.

Figura 5 - Relação comprimento total versus comprimento cauda para fêmeas e machos do $P$. subtilis na plataforma continental amazônica.

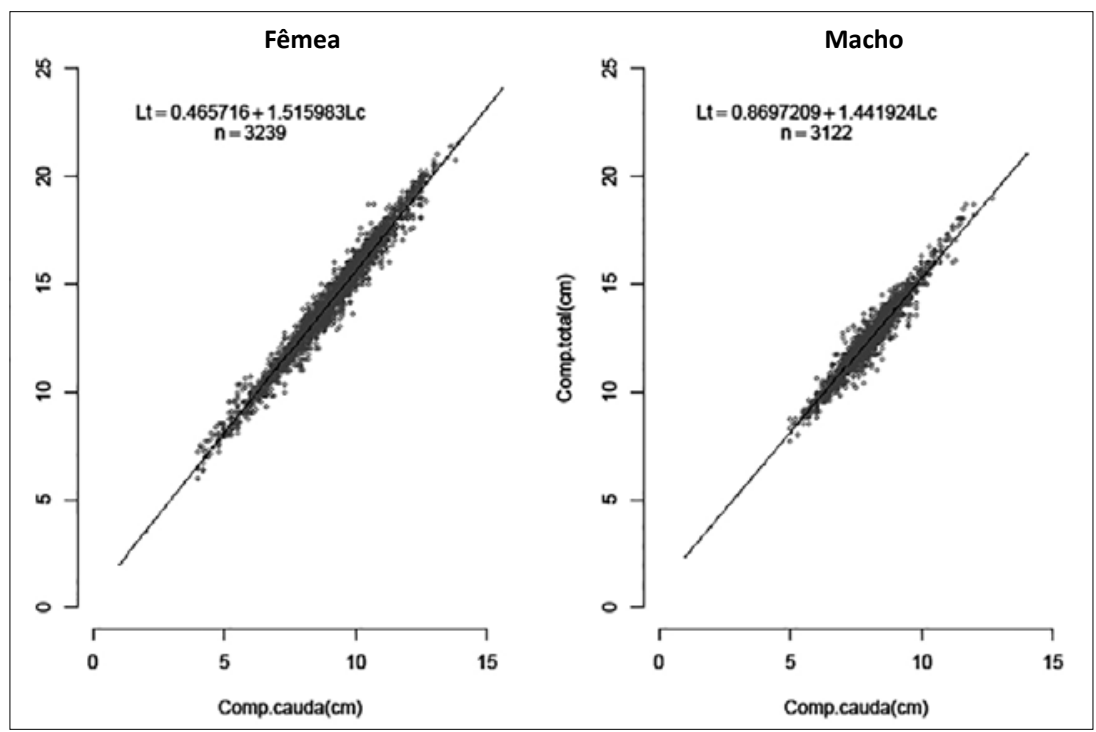




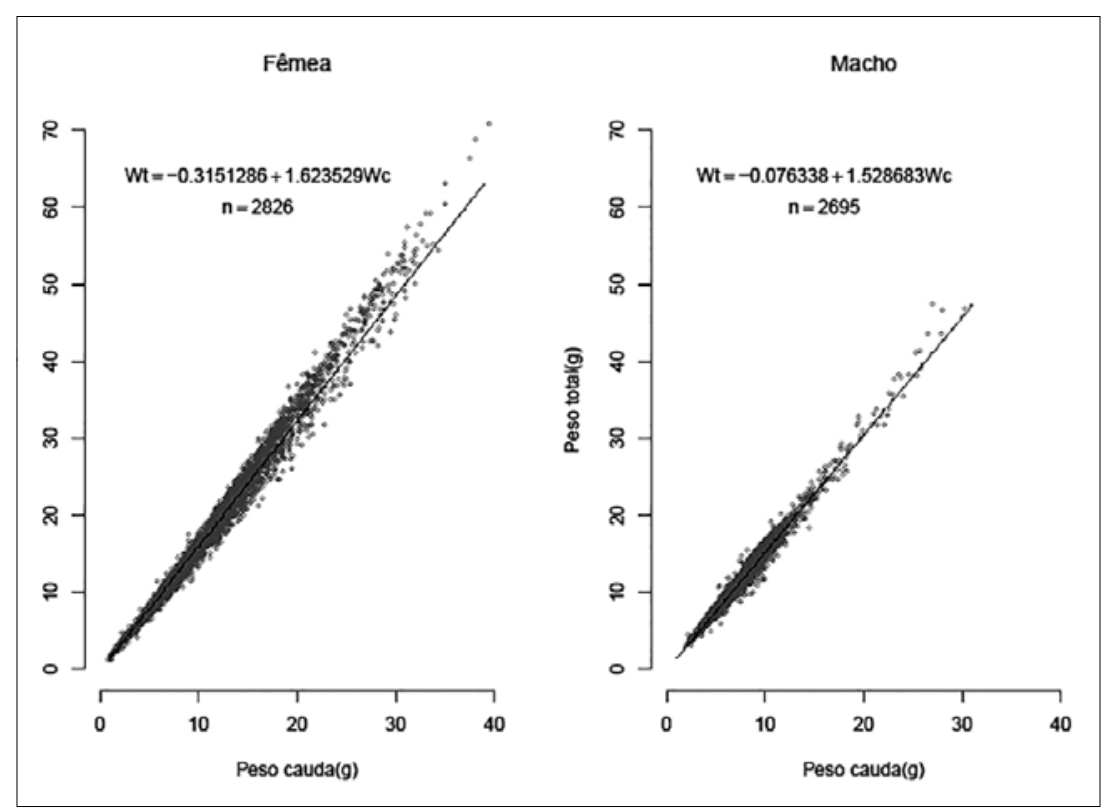

Figura 6 - Relação peso total versus peso cauda para fêmeas e machos do P. subtilis na plataforma continental amazônica

A título de ilustração e para comparação durante a discussão, são apresentados também na Tabela 8 os resultados obtidos por outros autores para as estimativas de parâmetros de regressões semelhantes. As estimativas dos parâmetros foram determinadas com as unidades de comprimento em milímetros. Encontram-se também na Tabela 9 as faixas de comprimento apresentadas por Barbosa \& Rocha (1977), onde se verifica que as faixas dos valores nas amostras por eles analisadas são bem mais estreitas que aquelas utilizadas no presente trabalho.

Tabela 8 - Parâmetros das relações peso total versus comprimento total e peso total versus peso de cauda do camarão-rosa da costa norte do Brasil, determinadas por outros autores e na presente análise.

\begin{tabular}{|c|c|c|c|c|c|c|}
\hline Relação & Sexo & $\mathbf{n}$ & $\mathbf{a}$ & b & $r$ & Autor \\
\hline \multirow{6}{*}{$\begin{array}{c}W t \sim L_{t} \\
\left(W_{t}=a^{*} L_{t}^{b}\right)\end{array}$} & Fêmea & 559 & 0,00000436 & 3,126 & 0,995 & BARBOSA e ROCHA(1977) \\
\hline & Fêmea & 326 & 0,00000400 & 3,146 & 0,990 & DRAGOVCHet al . (1980) \\
\hline & Fêmea & 2828 & 0,00000173 & 3,263 & $\left({ }^{*}\right)$ & Presente análise (Paulo Mbreira) \\
\hline & Macho & 519 & 0,00000667 & 3,024 & 0,994 & BARBOSA e ROCHA(1977) \\
\hline & Macho & 328 & 0,00000474 & 3,105 & 0,990 & DRAGOVCHet al. (1980) \\
\hline & Macho & 2684 & 0,00000356 & 3,111 & $\left({ }^{*}\right)$ & Presente análise (Paulo Mbreira) \\
\hline \multirow{4}{*}{$\begin{array}{c}W \mathrm{t} \sim \mathrm{Wc} \\
(\mathrm{Wt}=\mathrm{a}+\mathrm{bWc})\end{array}$} & Fêmea & 546 & $-0,133$ & 1,606 & 0,996 & BARBOSA e ROCHA (1977) \\
\hline & Fêmea & 2826 & $-0,315$ & 1,624 & $\left({ }^{*}\right)$ & Presente análise (Paulo Mbreira) \\
\hline & Macho & 493 & 0,044 & 1,515 & 0,998 & BARBOSA e ROCHA(1977) \\
\hline & Macho & 2695 & -0.076 & 1.529 & $\left({ }^{*}\right)$ & Presente análise (Paulo Mbreira) \\
\hline
\end{tabular}

Tabela 9 - Valores médios, máximos e mínimos das medidas biométricas de $P$. subtilis obtidos por Barbosa \& Rocha (1977) na costa Norte do Brasil.

\begin{tabular}{ccccccc}
\hline \multirow{2}{*}{ Medida } & \multicolumn{2}{c}{ Lt $(\mathbf{m m})$} & \multicolumn{2}{c}{ Wt $(\mathbf{g})$} & \multicolumn{2}{c}{ Wc $(\mathbf{g})$} \\
\cline { 2 - 7 } & Fêmeas & Machos & Fêmeas & Machos & Fêmeas & Machos \\
\hline Máximo & 18,0 & 14,8 & 56,2 & 25,4 & 32,3 & 16,0 \\
Médio & 13,2 & 12,1 & 20,9 & 13,9 & 13,1 & 9,1 \\
Mínimo & 6,4 & 5,0 & 2,2 & 1,0 & 1,4 & 0,6 \\
\hline
\end{tabular}


No caso do P. subtilis, em geral, fêmeas maiores apresentaram fator de condição superior em relação aos indivíduos menores, sendo menos expressiva no I trimestre, período em que predominam indivíduos pequenos. No caso dos machos, a tendência é semelhante, chegando a haver inclusive uma inversão do fator de condição no I trimestre, sendo maior para os indivíduos menores, conforme pode ser visto na Figura 7, onde os logaritmos dos valores do fator de condição trimestral por comprimentos são plotados contra os logaritmos dos comprimentos.

Verifica-se que o fator de condição para indivíduos maiores é sempre mais elevado, pois, ao atingirem maiores tamanhos, os indivíduos passam a crescer a uma taxa de crescimento menor, a acumular gordura e a desenvolver as gônadas, preparando-se para desova. Nota-se, também, que os valores para o fator de condição dos indivíduos maiores são mais elevados no segundo e terceiro trimestres do ano, sugerindo uma maior proporção de indivíduos maduros neste período. Outra observação que pode ser destacada é que no terceiro e quatro trimestres os valores para o fator de condição dos indivíduos maiores tendem a ser progressivamente menores.

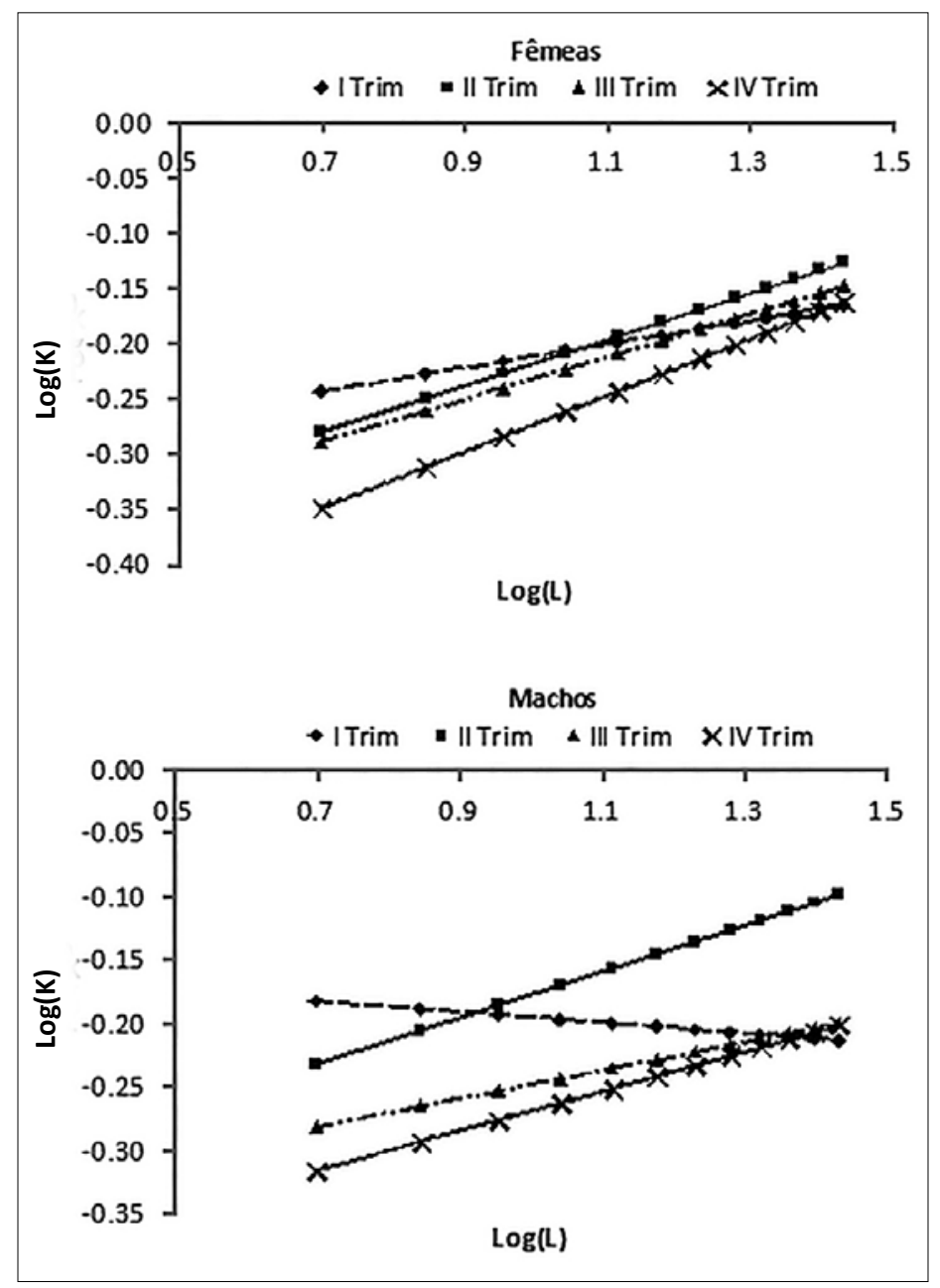

Figura 7 - Logaritmo do fator de condição trimestral versus comprimento para fêmeas e machos, calculado a partir da relação peso total versus comprimento total do $P$. subtilis na plataforma continental amazônica. 


\section{DISCUSSÃO}

Os valores médios encontrados para as medidas biométricas analisadas no presente trabalho, comprimento total $(L t)$, comprimento de cauda $(L c)$, peso total $(W t)$ e peso de cauda $(W c)$ (Tabela 2), são claramente maiores para fêmeas em relação aos machos, o que ficou confirmado também em resultados de testes " $t$ ", não apresentados aqui. Estes valores estão de acordo com aqueles obtidos por Barbosa \& Rocha (1977), porém, as faixas de valores mínimos e máximos apresentadas por estes autores para as mesmas variáveis foram de amplitude bem menor: Lt (fêmeas) entre 64,0 e 180,0 com média de 132,0 mm; Lt (machos) entre 50,0 e 148,0 com média de 121,2 mm; Wt (fêmeas) entre 2,2 e 56,2 com média de 20.9 g; Wt (machos) entre 1,0 e 25,4 com média de 13,9 g; Wc (fêmeas) entre 1,4 e 32,3 com média de 13,1 g; e Wc (machos) entre 0,6 e 16,0 com média de 9,1 g.

As maiores amplitudes de comprimentos e os maiores comprimentos médios verificados no presente trabalho, em comparação com aqueles indicados por Barbosa \& Rocha (1977), podem ser resultado do fato das amostras aqui consideradas terem sido obtidas em cruzeiros de pesquisa. Neste caso, a chance de se capturar indivíduos de uma faixa mais ampla de tamanhos é teoricamente maior, diferentemente do que ocorre na pesca comercial, onde as operações são direcionadas para áreas específicas, de maior abundância do recurso. Além do mais, as amostras de desembarques de capturas industriais aqui utilizadas eram compostas de um número bem superior de indivíduos.

As relações biométricas necessárias e mais frequentemente tomadas em estudos de organismos aquáticos, de forma geral, são o peso total versus o comprimento total e/ou furcal. No caso do $P$. subtilis da costa norte, onde praticamente todos os desembarques são compostos de indivíduos descabeçados, são necessárias relações que envolvam outras medidas de indivíduos inteiros e de caudas (ou abdômen). As relações peso total versus comprimento total, peso de cauda versus comprimento de cauda, peso total versus peso de cauda e comprimento total versus comprimento de cauda são especialmente úteis nos estudos de avaliação de estoques, quando não se dispõe de uma das medidas.

As relações entre peso e comprimento de peixes são bem estabelecidas biologicamente e tendem a ser não lineares. Isto pode ser entendido, intuitivamente, considerando-se que o comprimento é uma medida linear, enquanto o peso é uma medida relacionada ao volume do corpo (Ogle, 2009). Um aspecto importante nestas relações é que a variabilidade em peso aumenta à medida que o comprimento do peixe aumenta, ou seja, a variância sobre a curva de regressão é heterocedástica, conforme demonstra o aumento da dispersão dos pontos nos gráficos de dispersão e curvas ajustadas para as relações biométricas do camarão-rosa ( $P$. subtilis) no norte do Brasil (Figuras 3, 4, 5 e 6).

Um dos pressupostos básicos de regressões lineares, e também não lineares, é que a variância seja uniforme $\operatorname{var}\left(\varepsilon_{i}\right)=\sigma^{2}$, ao longo da faixa de variação dos valores das variáveis, ou seja, a variância deve ser homocedástica (Snedecor \& Cochran, 1967; Sokal \& Rohlf, 1995). As relações aqui avaliadas tendem a violar este pressuposto.

Geralmente, o modelo tipicamente utilizado para modelar a relação peso comprimento $\left(W=a L^{b} e^{\varepsilon}\right)$ é transformado para uma forma linear, aplicando-se logaritmo a ambos os lados da equação, $\log (W)=\log (a)+b * \log (L)+\log (\varepsilon)$, onde a variável resposta passa a ser $y=\log (W)$, a variável explanatória $x=\log (L)$, a declividade é igual a $b$ e interseção igual a $\log (a)$. Este procedimento, geralmente, torna o erro aditivo e estabiliza a variância sobre a linha de regressão, permitindo o ajuste pela técnica de regressão linear Tipo I, com base no método dos mínimos quadrados (Snedecor \& Cochran, 1967; Sokal \& Rohlf, 1995; Ogle, 2009). 
Porém, deve ser levado em conta que os valores estimados necessitam de ajustes na escala original uma vez que, neste tipo de procedimento, o valor estimado é a média do logaritmo do peso e o simples retro-cálculo para a escala original, por meio da exponenciação do logaritmo, resultará em subestimação do valor da variável resposta. Isto se deve ao fato da transformação do valor médio do logaritmo resultar na média geométrica dos valores na escala original, onde a média geométrica é menor que a média aritmética (Sampson, 2008; Ogle, 2009).

Várias alternativas para corrigir o vício no retro-cálculo do valor do peso médio, a partir do logaritmo, têm sido sugeridas na literatura. A mais comum é multiplicar o valor retro calculado pelo fator $e^{\mathrm{S}^{2} \times \mid \mathrm{y} / 2}$, derivado da análise das características da distribuição normal e da distribuição log-normal (Ogle, 2009). Porém, com as técnicas de computação hoje disponíveis, é mais recomendável utilizar a linearização apenas para estimar valores iniciais e calcular os parâmetros finais por meio da técnica não linear.

Vale salientar que as estimativas dos parâmetros calculadas por meio do modelo linear, com transformação logarítmica, numericamente não diferiram muito dos valores obtidos com o modelo não linear, utilizando-se a transformação Box-Cox. No entanto, segundo Ritz (2008), os intervalos de confiança dos parâmetros obtidos por meio da técnica não linear são mais estreitos.

Além da violação do pressuposto da homocedasticidade, é também comum que este tipo de variável apresente resíduos dos valores respostas não distribuídos normalmente. Esta característica fica claramente evidenciada por meio dos gráficos de resíduos, que apresentam uma clara tendência no formato de "corneta". Nestes casos, é frequentemente possível encontrar uma transformação que irá produzir valores repostas com distribuição de resíduos aproximadamente normais e variância constante. Para preservar a relação original entre as variáveis é necessário, porém, realizar a transformação em ambos os lados da equação, diferentemente do que se faz muitas vezes em regressão linear (Ritz \& Streibig, 2008).

Durante o cálculo das regressões, a exclusão de observações que apresentavam simultaneamente valores extremos de resíduos e distâncias de Cook não resultou, em geral, em diferenças importantes no ajuste da nova regressão. Talvez isto se deva ao fato das amostras serem suficientemente grandes, bem como ao fato da técnica dos mínimos quadrados ser robusta a pequenos desvios de normalidade.

É comum, em investigação pesqueira, se avaliar diferenças estatísticas entre parâmetros da regressão linear simples entre sexos, períodos, áreas, tratamentos, etc. (Barbosa \& Rocha, 1977; Leite \& Petrere-Júnior, 2006) utilizando-se o teste " $\mathrm{t}$ ". Neste trabalho, no entanto, a comparação das curvas das relações para dados agrupados entre fêmeas e machos foi feita por meio da estatística $F$, utilizando a técnica de "análise da soma dos quadrados dos resíduos (ARSS)", uma vez que a forte covariância entre os valores de $a$ e $b$, segundo Haddon (2011), invalidam esta análise. Ficou demonstrado que, em todos os casos, as curvas são simplesmente diferentes e, portanto, as conversões das medidas para a espécie têm que ser feitas utilizando-se, necessariamente, as equações determinadas para cada sexo.

O parâmetro $b$ é o expoente da forma aritmética da relação peso-comprimento e a declividade da linha de regressão na forma logarítmica. Teoricamente, se $b=3$, então indivíduos menores na amostra em consideração têm a mesma forma e condição dos indivíduos maiores. Se $b>3$, então indivíduos grandes cresceram mais em altura ou largura que em comprimento, seja como resultado de uma notável alteração ontogenética na forma do corpo, o que é raro, ou porque os indivíduos maiores na amostra eram mais 'gordos' 
(largos) que os indivíduos menores, o que é comum. Por outro lado, se $b<3$, então indivíduos maiores sofreram alterações no formato do corpo para ficar mais alongados ou então os indivíduos menores estavam em melhores condições nutricionais no período da amostragem. Em geral, há uma tênue, mas significativa tendência para a maioria dos peixes se tornarem mais 'gordos' (largos) à medida que crescem (Froese, 2006).

Quando se discute o expoente $b$ de uma única relação peso-comprimento, porém, devem ser avaliadas apenas diferenças na condição entre indivíduos pequenos e grandes na respectiva área, para determinado período de tempo. Somente quando estimativas de parâmetros das relações peso-comprimento, cobrindo de forma razoável as variações geográficas, sazonais e interanuais, estiverem disponíveis faz sentido discutir crescimento isométrico versus alométrico de espécies como um todo, baseado numa estimativa média de $b$, uma vez que a condição média dos indivíduos varia entre estações, localidades e anos, resultando em diferenças nas relações peso-comprimento. Portanto, como padrões de crescimento alométrico acentuado são raros, as alterações deveriam ser analisadas considerando o que ocorre nas diferentes fases de crescimento, com uma discussão dos benefícios evolucionários associados com tal mudança ontogenética nas proporções do corpo (Froese, 2006).

O parâmetro $a$ é o coeficiente da forma aritmética da relação peso-comprimento e a interseção da forma logarítmica, havendo uma forte inter-relação entre os parâmetros $a$ e $b$ da regressão peso-comprimento. Sempre que o valor estimado de $b$ diminui aumenta o valor estimado de $a$ e vice-versa (Ricker, 1975), sendo o parâmetro $a$ relacionado ao fator de condição.

Comparando as equações das relações peso total versus comprimento total (Wt $x$ Lt) e peso de cauda versus comprimento de cauda (Wc $x$ Lc) determinadas nesta análise com outras semelhantes, apresentadas nos trabalhos de Barbosa \& Rocha (1977) e Dragovich et al. (1980), já apresentadas previamente na Tabela 9, verifica-se que há uma diferença expressiva entre os parâmetros das mesmas, embora não seja possível a aplicação de testes estatísticos comparativos. Talvez haja alguma explicação biológica para este fato, mas não se dispõe de informações e dados que permitam alguma inferência.

Deve-se notar, no entanto, que as amostras utilizadas na presente análise são de tamanho consideravelmente maior que aquelas utilizadas nos citados trabalhos, cobrindo uma faixa bem ampla dos valores das variáveis e foram obtidas ao longo de todos os meses do ano. Ressalte-se ainda que, embora não sejam mostradas aqui, regressões calculadas com os dados separados das amostras dos cruzeiros de pesquisa e dos desembarques não apresentaram diferenças, com as curvas se sobrepondo indistintamente.

Por outro lado, o processo de mensuração das variáveis, utilizado por aqueles autores, não foi descrito, especialmente a forma de obtenção do peso dos indivíduos, não sendo possível comparar razões para eventuais diferenças, devendo ser levado em conta a maior precisão das balanças hoje utilizadas. Outro aspecto importante é que, em geral, existem diferentes estágios de crescimento nas diversas fases de vida da espécie e, no caso de Barbosa \& Rocha (1977), foram utilizados dados coletados da fase de juvenil no estuário o que pode ter influenciado a estimação dos parâmetros das regressões.

No caso específico das relações peso-comprimento, utilizadas para estimação do peso correspondente de um dado tamanho de indivíduo capturado pela frota industrial, as regressões calculadas com os dados das faixas de comprimentos correspondentes a estas capturas permitirá prever melhor os respectivos pesos, uma vez que nos estádios iniciais os peixes crescem em comprimento a uma taxa maior que em outras dimensões e, assim, a proporção peso-comprimento difere daquela obtida para indivíduos maiores. (Froese, 2006). 
Em relação ao fator de condição, os valores sempre superiores observados para os indivíduos maiores, em relação aos indivíduos menores, sugerem a presença de indivíduos maduros ao longo de todo o ano, mas em menor proporção no primeiro trimestre, quando é maior a predominância de indivíduos jovens, de menor tamanho médio (Aragão, 2012). Isto significaria também que, ao longo do ano, à medida que os indivíduos vão atingindo maiores tamanhos e vão amadurecendo, a taxa de crescimento diminui, e passam a acumular gordura e a desenvolver as gônadas, tendo o aumento do peso da gônada contribuição importante na elevação do fator de condição.

Os valores mais elevados para o fator de condição dos indivíduos maiores no segundo e terceiro trimestres, bem como a tendência dos valores do fator de condição serem progressivamente menores entre o terceiro e quarto trimestres, sugerem a presença de uma maior proporção de indivíduos maduros no segundo e terceiro trimestre, que corresponde ao período de desova mais intensa (Aragão, 2012), observando-se também uma sequencial diminuição do número de indivíduos prontos para desovar nos dois períodos seguintes.

As conclusões acima para o $P$. subtilis mantêm coerência com as observações de que peixes em períodos anteriores aos distúrbios reprodutivos começam a apresentar marcados desvios da "lei do cubo". Embora seja indicado na literatura que ocorre acentuada perda de peso imediatamente após a desova, parece que é rapidamente revertida (Froese, 2006).

\section{CONCLUSÕES}

- As equações aqui determinadas descrevem convenientemente as relações entre as diferentes variáveis, o que fica objetivamente demonstrado através dos gráficos dos valores preditos contra os valores observados e as curvas para todas as relações, em bases anuais, e também as medidas biométricas, apresentam diferenças altamente significativas entre machos e fêmeas;

- As diferenças entre as relações "peso total versus comprimento total" e "peso total versus peso de cauda" calculadas por outros autores pode ter relação com o fato das amostras aqui utilizadas serem expressivamente maiores e cobrirem uma faixa bem mais ampla na escala temporal e espacial, bem como aos procedimentos e instrumentos de mensuração utilizados. A incorporação de dados de juvenis do estuário no cálculo de algumas relações por outros autores também pode ter contribuído para as diferenças;

- Os valores sempre superiores observados para o fator de condição dos indivíduos maiores sugerem a presença de indivíduos maduros ao longo de todo o ano, mas em menor proporção no primeiro trimestre, devido a predominância de indivíduos jovens. Sugere ainda que, à medida que os indivíduos vão atingindo maiores tamanhos e amadurecendo, a taxa de crescimento diminui, passando a acumular gordura e a desenvolver as gônadas, tendo o peso destas contribuição importante na elevação do fator de condição.

\section{REFERÊNCIAS BIBLIOGRÁFICAS}

Aragão, J. A. N., 2012. Dinâmica populacional e avaliação do estoque do camarão rosa (Farfantepenaeus subtilis Pérez Farfante, 1967) na plataforma continental amazônica brasileira. Tese de Doutorado, Programa de Pós Graduação em Ciências da Engenharia Ambiental, Escola de Engenharia de São Carlos, Universidade de São Paulo, 243 p., São Carlos, 2012. 
Aragão, J. A. N.; Cintra, I. H. A. \& Silva, K. C. A. Revisão dos dados de esforço de pesca e captura das pescarias industriais de camarão-rosa, Farfantepenaeus subtilis (Pérez Farfante, 1967) (Crustacea, Decapoda, Penaeidae) na região norte do Brasil. Bol. Téc. Cient. Cepnor, Belém, v. 4, n. 1, p.31-44, 2004.

Aragão, J. A. N.; Silva, K. C. A. \& Cintra, I. H. A. A pesca industrial do camarão na costa norte, p.51-54, in Oliveira, G.M. (ed.), Pesca e aqüicultura no Brasil, 1991/2000: produção e balança comercial. Ibama, 260 p., Brasília, 2005.

Aragão, J. A. N.; Cintra, I. H. A.; Silva, K. C. A. \& Vieira, I. J. A. A explotação camaroeira na costa norte do Brasil. Bol. Téc. Cient. Cepnor, Belém, v. 1, n. 1, p.7-32, 2001.

Barbosa, F. I. \& Rocha, J. M. Algumas relações biométricas para o camarão-rosa (Penaeus aztecus subtilis Pérez-Farfante, 1967) da costa norte do Brasil. SUDEPE/PDP, 12 p., Brasília, 1977.

Crawler, M. J. The R book. John Wiley \& Sons Ltd, 949 p., West Sussex, 2007.

Dragovich, A.; Jones, A.C. \& Boucher, G.C. United States shrimp surveys off the Guyana and Northern Brazil (1972-1976). In Jones, A.C. \& Villegas, L. Proceedings of the Working group on shrimp fisheries of northeastern South America. WECAFC Rep. p.1-46, Panama City, 1980.

Fischer, W. FAO species catalogue identification sheets fishery purposes. FAO, v. 6, p.2-21, Roma, 1978.

Fox, J. Nonlinear regression and nonlinear least squares. 2002. Disponível em: http://cran.r-project.org/doc/contrib/Fox-Companion/appendix-nonlinear-regression.pdf. Acesso em: 14 mar. 2010.

Froese, R. Cube law, condition factor and weight-length relationships: history, meta-analysis and recommendations. J. Appl. Ichthyol., v. 22, p.241-253, 2006.

Haddon, M. Modelling and quantitative methods in fisheries. Chapmam \& Hall/CRC, 405 p., New York, 2011.

Holthuis, L. B. FAO species catalogue; shrimps and prawns of the world. An annotated catalogue of species of interest to fisheries. FAO, 271 p., Rome, 1980.

IBAMA. Camarão norte e piramutaba: relatórios reuniões dos Grupos Permanentes de Estudos. Instituto Brasileiro do Meio Ambiente e dos Recursos Naturais Renováveis. Coleção Meio Ambiente: Série Estudos-Pesca 9, 148 p., Brasília, 1994.

Leite Júnior, N. O. \& Petrere Júnior, M. Growth and mortalities of the pink-shrimp Farfantepenaeus brasiliensis Latreille, 1970 and F. paulensis Pérez-Farfante 1967 in Southeast Brazil. Braz. J. Biol., v. 66, n. 2, p.523-536, 2006.

Ogle, D. H. Length-weight relationship analysis Vignette. 2009. Disponível em: http:// www.ncfaculty.net/dogle/fishR/gnrlex/LengthWeight/LengthWeight.pdf. Acesso em: 17 jun. 2009.

$\mathrm{R}$ Development Core Team R: A language and environment for statistical computing. $\mathrm{R}$ Foundation for Statistical Computing, Vienna, Austria. 2009. Disponível em: http:// www.R-project.org. Acesso em: 07 jan. 2011.

Ragonese, S.; Bertolino, F. \& Bianchini, M.L. Biometric relationships of the red shrimp, Aristaeomorpha foliacea Risso 1827, in the Strait of Sicily (Mediterranean Sea). Sci. Mar., v. 61, n. 3, p.367-377, 1987.

Ricker, W. E. Computation and interpretation of biological statistics of fish populations. Bulletin of the Fisheries Research Board of Canada, 382 p., Ottawa, 1975 
Ritz, C. \& Streibig, J.C. Nonlinear regression with R. Springer, 148 p., New York, 2008.

Sampson, D. Dynamics of Marine Biological Resources. 2008. Disponível em: http:/ / oregonstate.edu/instruct/fw431/sampson/. Acesso em: 23 set. 2008.

Snedecor, G.W. \& Cochran, W.G. Statistical Methods. Ames: The Iowa State University Press. 593 p., Iowa, 1967.

Sokal, R. R. \& Rohlf, F. J. Biometry: the principles and practice of statistics in biological research. W. H. Freeman and Company, 887 p., New York, 1995.

Takeda, M. Crustaceans and mollusks trawled off Suriname and French Guiana. Japan Marine Fishery Resoure Research Center, 354 p., Tóquio, 1983.

Venables, W. N. \& Simth, D. M. An Introduction to R. Copyright @1999-2006 R. Development Core Team, 103 p., 2008.

Voss, G. L. A key to the commercial and potentially commercial shrimp of the family Penaeidae of the western north Atlantic and the Gulf of Mexico. Florida State Board Conservation Marine Laboratory, Tech. Ser. No. 14. 22 p., 1955.

Williams, A. B. Shrimps, lobsters, and crabs of the Atlantic coast of the Eastern United States, Maine to Florida. Smithsonian Institution Press, 550 p., Washington, 1984. 